\title{
Reduction of the elevator illusion from continued hypergravity exposure and visual error-corrective feedback
}

\author{
ROBERT B. WELCH, MALCOLM M. COHEN, and CHARLES W. DEROSHIA \\ NASA-Ames Research Center, Moffett Field, Califormia
}

\begin{abstract}
Ten subjects served as their own controls in two conditions of continuous, centrifugally produced hypergravity $(+2 \mathrm{Gz})$ and a 1-G control condition. Before and after exposure, open-loop measures were obtained of (1) motor control, (2) visual localization, and (3) hand-eye coordination. During exposure in the visual feedback/hypergravity condition, subjects received terminal visual error-corrective feedback from their target pointing, and in the no-visual feedback/hypergravity condition they pointed open loop. As expected, the motor control measures for both experimental conditions revealed very short lived underreaching (the muscle-loading effect) at the outset of hypergravity and an equally transient negative aftereffect on returning to $1 \mathrm{G}$. The substantial (approximately $17^{\circ}$ ) initial elevator illusion experienced in both hypergravity conditions declined over the course of the exposure period, whether or not visual feedback was provided. This effect was tentatively attributed to habituation of the otoliths. Visual feedback produced a smaller additional decrement and a postexposure negative aftereffect, possible evidence for visual recalibration. Surprisingly, the target-pointing error made during hypergravity in the no-visual-feedback condition was substantially less than that predicted by subjects' elevator illusion. This finding calls into question the neural outflow model as a complete explanation of this illusion.
\end{abstract}

Until the advent of high-speed vehicles (trains, automobiles, airplanes, spacecraft), human beings had evolved and spent their entire lives in a nearly constant gravitational force field. It is not surprising, therefore, that exposure to sudden changes in this force can seriously disrupt perception and perceptual-motor coordination. For example, rapid forward acceleration ( $+\mathrm{Gx}$ ) in a jet plane causes a dramatic apparent rise in the visual field (the oculogravic illusion; Graybiel, 1952) and a feeling that the body has been pitched backward (the posturogravic illusion; Clark \& Graybiel, 1949). Occasionally, pilots in airplanes being catapulted from aircraft carriers accept these powerful illusions as real and some have died as a consequence as they attempted to compensate for the apparent excessive noseup attitude of the aircraft, only to dive into the ocean (Cohen, 1981). Still another visual-vestibular effect is the oculogyral illusion, in which bodily rotation causes an ap-

This research was supported by the National Aeronautics and Space Administration (Project UPN 199-16-12-34). The authors wish to thank $K$. Traicoff for her design and construction of the electronic interface that allowed for precise measurement of subjects' visual, motor, and visual-motor responses. We are also indebted to $\mathrm{G}$. Buhtz for designing our data acquisition program, R. Pelligra and his assistants, D. Peelor and D. English, who monitored the physical well-being of subjects, and G. Mulenberg and D. Gundo, who directed centrifuge operations. Finally, thanks go to N. G. Daunton, S. M. Ebenholtz, R. B. Post, A. Stoper, D. L. Tomko, and the anonymous reviewers for their helpful comments on earlier drafts of this manuscript. Correspondence should be addressed to R. B. Welch, NASA-Ames Research Center, Mail Code 23911, Moffett Field, CA 94035-1000 (e-mail: robert_welch@qmgate. arc.nasa.gov). parent spinning of the observer's visual field (e.g., Graybiel \& Hupp, 1946).

In reduced $\mathrm{G}$ (hypogravity), visual objects in an otherwise dark setting appear lower than they are (Graybiel, Clark, \& MacCorquodale, 1947), and initial attempts to reach for them are generally too high (von Beckh, 1959). ${ }^{1}$ In hypergravity along the head-to-foot $(+\mathrm{Gz})$ axis, the effects tend to be just the opposite. Thus, objects appear too high, a phenomenon known as the "elevator illusion" (Cohen, 1973; Correia, Hixson, \& Niven, 1968; Shöne, 1964; Whiteside, 1961), and initial reaches are too lowthe "muscle-loading effect" (Cohen, 1970a, 1970b; Cohen \& Welch, 1988).

The return to normal gravity after extended exposure to hyper- or hypogravity is frequently accompanied by "negative aftereffects," evidence that some form of adaptive recalibration has occurred. Cohen (1970b), for example, found that reaching during about $5 \mathrm{~min}$ of exposure to $+2 \mathrm{Gz}$ was followed in $1 \mathrm{G}$ by a transient tendency to reach too high.

The most popular explanation of the elevator illusion is the neural outflow model (Cohen, 1973; Whiteside, Graybiel, \& Niven, 1965). According to this notion, visual objects appear to be higher than they are because the reflexive innervation of the ocular muscles during Gz hypergravity that serves to rotate the eyes downward (see, e.g., Marcus \& Van Holten, 1990) is being countered by voluntary efference for the upward eye movements that are necessary to maintain fixation of the target. Thus, because in normal gravity this is the pattern of neural outflow used to fixate an object that is higher than the current target, this is where that target now appears to be located. 
It is likely that the muscle-loading effect results from the observer's initial failure to exert the effort required to overcome the unexpected extra weight of the arm. Because this underreaching is presumably based on inappropriate neural outflow (efference) with respect to desired action, it should be measured in a manner that reduces as much as possible the role of visual and proprioceptivekinesthetic feedback (afference). One attempt to satisfy this requirement is to instruct subjects to reach ballistically at unseen (or imagined) targets. Crucial to the aim of the present experiment is the fact that the muscle-loading effect is very quickly overcome by an adaptive process that does not require visual feedback, but that can occur on the basis of proprioceptive-kinesthetic information alone (Cohen, 1970a, 1970b; Cohen \& Welch, 1992). ${ }^{2}$

Because the elevator illusion and muscle-loading effect work in opposite directions and thus tend to negate each other, it is possible for initial target-pointing attempts during hypergravity to be relatively accurate. However, after the muscle-loading effect has been eliminated by means of proprioceptive-kinesthetic feedback from the limb, subjects will tend to point (still open loop) too high at visual targets because the latter continue to be influenced by the elevator illusion, which remains at full strength (Cohen, 1970a, 1970b; Cohen \& Welch, 1992). Assuming that visual feedback continues to be denied (and that no spontaneous decay of the elevator illusion or change in felt limb position has occurred), overreaching should persist indefinitely because under these conditions there is no way for observers to discover that they are pointing too high.

There appears to be no difference between pilots and nonpilots in the reported strength of their oculogravic illusions (see, e.g., Cohen, Crosbie, \& Blackburn, 1973), a finding suggesting that this and perhaps other visualvestibular illusions are impervious to experience. This may be a premature conclusion, however, because it is far from obvious that mere passive exposure to or intellectual knowledge about an illusion can influence it. On the other hand, it is possible that a change might occur if observers are allowed to explore the illusory stimulus physically and receive sensory feedback from their efforts. We term this notion the "interaction hypothesis."

Empirical support for this hypothesis comes from three disparate research areas. First, it has been demonstrated that certain geometric visual illusions (e.g., Mueller-Lyer, Poggendorf) are greatly weakened or eliminated after observers have scanned them saccadically and learned to correct for their initial fixation errors (Day, 1962; Festinger, White, \& Allyn, 1968; Hoenig, 1972). Similarly, Kilpatrick (1954) reported that subjects who actively explored the "Ames Distorted Room" by poking around in it with a rod eventually came to perceive its true configuration. Finally, Kalil and Freedman (1966) and Uhlarik and Canon (1971) demonstrated that if observers whose visual fields were prismatically displaced were directed to point at targets and provided with terminal visual feedback about their errors, their judgments of visual straight ahead became more accurate.
On the basis of the preceding evidence, we predicted that the elevator illusion would be attenuated if subjects were allowed to interact with it by means of hand-eye coordination.

\section{METHOD}

\section{Subjects}

The sample consisted of 9 males and 1 female (mean age $=34.9$ years), drawn from the local community and from employees at NASA-Ames Research Center. All subjects were naive to the purposes of the experiment and had passed a physical examination that, when deemed necessary, included a treadmill EKG test. Naturally, subjects were informed of the potential risks of the experiment and allowed to withdraw from it if they wished; several chose to do so. Of the original sample of potential subjects, 2 were replaced, 1 after an initial familiarization run in the centrifuge had revealed orthostatic intolerance to hypergravity and the other for failing to demonstrate a reliable elevator illusion.

\section{Safety Precautions}

Prior to the experiment, subjects underwent one or more familiarization runs, during which they experienced periods of hypergravity, ranging from $+1.25 \mathrm{Gz}$ to $+2.0 \mathrm{Gz}$, and practiced the experimental tasks. In addition, measures of their susceptibility to the elevator illusion were obtained.

Brief exposures to $+2.0 \mathrm{Gz}$ are generally considered benign for healthy individuals. However, because of our desire to use an extended period of exposure to this $\mathrm{G}$ level and to require subjects to engage simultaneously in physical activity, certain precautions were taken. On all centrifuge runs, subjects' heart rate and EKG were continually monitored by a physician or medical technician, who sat in the control room adjacent to the centrifuge rotunda. In addition, for those subjects with no previous centrifugation experience, a Doppler ultrasonic sensing system was used to monitor temporal artery blood flow as a means of detecting potential orthostatic intolerance. Further, a TV screen presented to the medical monitor a close-up view of the subject's face that was provided by a TV camera attached to the subject's chair in the centrifuge cab. Because the cab was to be in complete or nearly complete darkness during the experiment, illumination for the camera was provided by an infrared light source, located beside the TV camera. Finally, subjects could terminate a centrifuge run at any time by releasing a normally open switch attached to the left armrest of the chair.

The medical monitor was prepared to stop the centrifuge upon the subject's request and/or any evidence of physical or psychological stress, as indicated by the physiological measures or the subject's visual appearance on the TV monitor. Such events occurred on only 4 (out of 50) centrifuge runs, not including familiarization sessions.

\section{The Centrifuge}

The experiment took place in the NASA-Ames Research Center 20-G centrifuge. Mounted at one end of the centrifuge arm was a "light-tight" cab, $2.1 \mathrm{~m}$ high $\times 1.8 \mathrm{~m}$ wide $\times 2.3 \mathrm{~m}$ deep (radial direction). The distance between the center of rotation and the center of the cab was $7.62 \mathrm{~m}$. Operating controls, instrumentation displays, and data acquisition equipment were located in the control room. The subject's chair, which, in the present experiment, faced away from the center of rotation, was inside the cab. A single-axis gimbal allowed the precisely balanced chair to remain aligned with the changing gravitational-inertial vector as the centrifuge accelerated to the desired rotation rate. When this rate was attained, a brake was activated to prevent the chair from pitching (which can be quite nauseogenic). Thus, centrifugation causes an increase in the subject's body weight while he/she remains upright with respect to the gravitationalinertial force vector. During an experiment, all communication be- 
tween the subject and research and medical personnel was effected through an intercom system.

\section{Design}

Subjects served as their own controls in two hypergravity conditions, referred to as visual feedback and no visual feedback, each of which was replicated in a separate testing session. Each condition and replication occurred on separate days, their order was counterbalanced by means of an $\mathrm{ABBA}$ or $\mathrm{BAAB}$ scheme $(\mathrm{A}=$ feedback; $\mathrm{B}=$ no feedback). The interval between any two sessions varied, but was usually 1 week. Hypergravity exposure (perexposure) averaged $26.8 \mathrm{~min}$ and was bracketed by short pre- and postexposure phases of normal gravity. The length of the perexposure phase ranged from 21.4 to $31.3 \mathrm{~min}$, depending on a given subject's response rate. Finally, subjects participated in a 1-G control condition, usually on a day subsequent to the last hypergravity session. Aside from $\mathrm{G}$ level, the procedure and stimulus situation for this condition were identical to those of the visualfeedback condition. The aim of the control condition was to assess possible changes in any of the measures that might occur as the result of such factors as repeated testing, fatigue, and drifting attention.

\section{Procedure and Apparatus}

After being instrumented for the neurophysiological measures and receiving last-minute instructions, the subject sat in the centrifuge chair. Straps were used to secure his/her trunk, legs, and left arm to the chair (the left hand remained free to operate the emergency switch). Head movements were constrained by means of a modified football helmet worn by the subject and bolted to the back of the chair. Visual horizon was equated for all subjects by raising or lowering the chair, as necessary, to a designated height. Attached to the tip of the subject's right index finger by means of rubber and latex was a metal sensor that detected reaching accuracy when the finger made contact with the curved, vertically aligned screen directly in front of the subject.

During the 1-G pre- and postexposure phases of a given hypergravity session (or of the 1-G control condition), subjects (1) pointed at the (unseen) screen at a level that they perceived to be at shoulder height in the dark (motor control response), (2) used a toggle switch to set a movable spot of light (projected on the screen) to the horizon for a period of $1 \mathrm{~min}$ (visual localization response), and (3) pointed ballistically at visual targets (hand-eye coordination response) on the screen. Subjects received no visual or verbal feedback about their performance on any of these pre- and postexposure measures, all of which occurred while the centrifuge was rotating at a "resting rate" of $3 \mathrm{rpm}$ (producing an imperceptible .003-Gz increase in gravitational-inertial force). There follows a more detailed description of the three dependent measures and the pre-, per-, and postexposure phases of the experiment.

Motor control response (shoulder-pointing). For the motor control measure, subjects in the completely darkened centrifuge cab reached out with the right hand as quickly as possible and touched the unseen screen at the position they felt to be directly opposite the top of the right shoulder. These "shoulder-pointing responses" were assumed to represent a relatively "pure" measure of motor control (i.e., neural outflow) because they were performed ballistically and without vision. Ten measures were obtained (one every $3 \mathrm{sec}$ ) during the preexposure phase. Subjects initiated a response at the sound of a tone and withdrew the finger from the screen upon hearing a tone of a different frequency. Each response began with the hand located at the end of the right armrest. If the finger was inadvertently moved before its position could be recorded, a computer-controlled auditory "razz" sounded and the response was repeated.

Visual localization response (visual horizon setting). Visual localization was measured by having subjects use a hand-held toggle switch, located at the end of the right armrest of the chair, to control the vertical position of a spot of light so that it appeared to be at their (imagined) visual horizon in the otherwise dark centrifuge cab. The horizon was defined for them as "where the sky would appear to meet the water if you were on the beach, looking out over the ocean." The visual stimulus was a $.5^{\circ}$ spot of red light projected onto the screen by a $.5-\mathrm{mW}$ helium-neon laser-pointer and controlled by a motor. The spot was constantly driven upward by the motor and the subject's task was to counteract this motion so that it remained at apparent horizon until he/she was instructed to release the switch. (Preliminary observations indicated that dynamic stimulus tracking was preferable to merely setting an otherwise stationary spot to the horizon because the former procedure kept subjects alert throughout the entire 60 -sec measurement period and ready to reposition the stimulus if necessary.) The experimenter waited $10 \mathrm{sec}(60 \mathrm{sec}$ on the first perexposure test) after the subject first reported the spot to be at the horizon before commencing a $60-\mathrm{sec}$ recording period $(2-\mathrm{Hz}$ sampling rate). Previous research (e.g., Cohen, 1973) has indicated that during the early stages of hypergravity exposure, many observers, after reporting the spot to be at the horizon, nevertheless continue to adjust its position in the direction congruent with an increase in the strength of the illusion.

Hand-eye coordination response (target pointing). Hand-eye coordination was measured by having the subject point ballistically at three targets, each consisting of a tightly bunched horizontal triad of red light-emitting diodes (LEDs), $.32 \mathrm{~cm}$ in diameter, imbedded in the screen. The distance of the three targets from the subject's nasum was $58 \mathrm{~cm}$. The vertical locations of the top and middle targets were, respectively, $16.2 \mathrm{~cm}$ and $6.2 \mathrm{~cm}$ above true eye level, and the bottom one was $3.8 \mathrm{~cm}$ below eye level. This displacement of the three targets toward the upper part of the screen served as a precaution against the possibility that initial target-reaching responses would be so low, due to the muscle-loading effect, that reaches for the lowest target would miss the screen altogether.

Subjects pointed 15 times, 5 at each of the three targets. (Actually, they were instructed to point to the left of the targets to avoid damaging them with the metal sensor.) The order of pointing was pseudorandomized and intertrial interval averaged $2.3 \mathrm{sec}$. The hand departed from the armrest at the appearance of the target, which was then extinguished for the remainder of the reaching response. Although subjects were thus forced to point at remembered target position, the interval between its disappearance and completion of the response was so short (a fraction of a second) that it is unlikely that reaching accuracy was compromised by this procedure.

After the last of the preexposure measures, the centrifuge was smoothly accelerated over a $30-\mathrm{sec}$ period to a constant rotation rate of 14.5 RPM, which produced a $Z$-axis accelerometer reading of exactly +2.0 . Next, subjects repeated the open-loop motor control $(20$ trials), visual localization ( $1 \mathrm{~min}$ ), and hand-eye coordination (15 trials) responses, in that order. For most of the remainder of the perexposure phase, subjects pointed at the targets, either with visual error-corrective feedback (visual-feedback condition) or without it (no-visual-feedback condition). After every block of 30 such responses, they set the spot of light to the apparent horizon (without feedback) for a period of $1 \mathrm{~min}$. The interval between each of these perexposure tests was approximately $4.5 \mathrm{~min}$.

Visual feedback was provided for subjects in the visual-feedback condition by allowing them to see their pointing finger for $2 \mathrm{sec}$ at the termination of each target-pointing response. The finger was made visible by back illuminating it with LEDs that were attached to its ventral surface. Subjects were instructed to keep the finger motionless against the screen for the $2 \mathrm{sec}$ that they could see it and to use accuracy on a given trial as the basis for subsequent performance. In the no-visual-feedback condition, the finger could not be seen at any time during the target-pointing response.

Just before the end of the perexposure phase, subjects in both conditions were measured on target-pointing accuracy without visual feedback ( 15 trials) and on shoulder pointing ( 10 trials), in that order. Then the centrifuge was decelerated ( 30 -sec ramp-down period) to $3 \mathrm{RPM}$, in readiness for the postexposure phase, during which the three response measures were obtained in the same manner and order as in the preexposure phase. 


\section{RESULTS AND DISCUSSION}

\section{An Overview of Predicted Results}

Subjects' visual localization responses were expected to indicate that the spot of light appeared substantially higher than its true location (i.e., the elevator illusion). However, because of the doubled weight of the arm, subjects' initial hand-eye coordination (target pointing) would presumably not have been in error by the same amount as the elevator illusion. To avoid this possibility, subjects were not allowed to point at the visual target until the muscleloading effect had been eliminated by adaptation (after having been measured). Finally, according to the interaction hypothesis, the hypergravity condition that included visual error-corrective feedback, in comparison to the nofeedback control condition, should have resulted in a reduced elevator illusion.

\section{Motor Control Response (Shoulder Pointing)}

The results of the motor control measure are depicted in Figure 1. We did not anticipate any difference between the visual feedback and no-visual-feedback conditions on this measure, an expectation confirmed by visual inspection of the data. As a consequence, the results for the two hypergravity conditions have been combined. Depicted in the figure is the shoulder-pointing response for each of the first 10 trials of the 20 -trial set that occurred at the begin- ning of the perexposure period. This is followed by the mean (A) of the last 10 trials of this set, the mean (B) of the 10 measures taken at the end of the perexposure phase, and finally the response on each of the 10 postexposure trials. All scores have been normalized by subtracting them from their associated preexposure scores. (For this and the other dependent variables, preexposure accuracy was essentially the same for all conditions.)

The underreaching observed on Perexposure Trial 1 for the combined 2-G conditions (but not the 1-G control) was the muscle-loading effect. This extremely transient effect was followed by an unexpected overcompensatory response that, even more surprisingly, occurred for the 1-G condition as well. Nevertheless, it would appear that, by the end of the initial 20 perexposure trials (mean B), subjects in the combined 2-G conditions had overcome most, if not all, of the muscle-loading effect, allowing the elevator illusion to hold full sway over subsequent visual target-pointing accuracy.

The overreaching observed for the 2-G conditions on the first two postexposure trials represents the negative aftereffect (muscle unloading) and was followed by substantial underreaching, probably due to arm fatigue.

\section{Visual Localization Response (Horizon Setting)}

Figure 2 presents the mean per- and postexposure settings of visual horizon for the three conditions, normal-

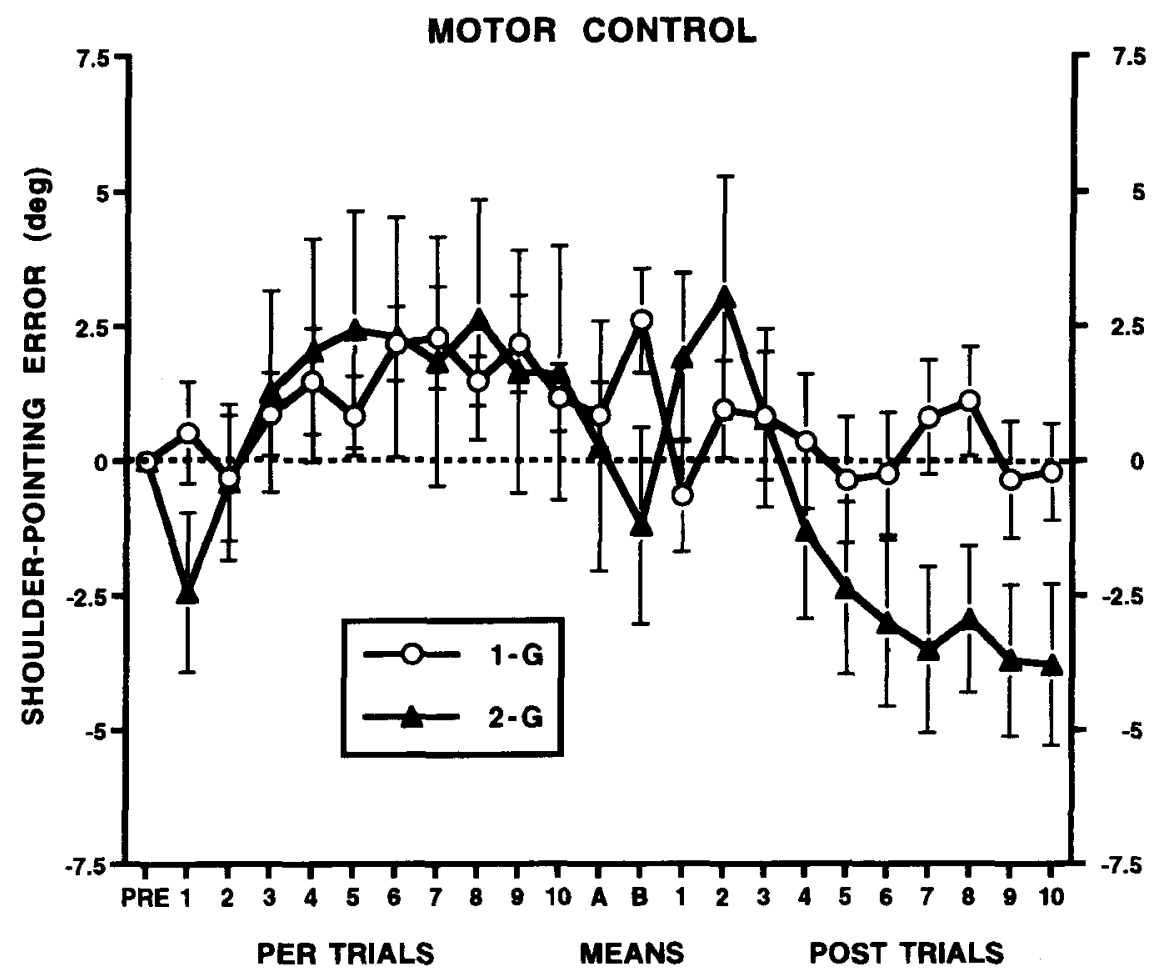

Figure 1. Shoulder-pointing errors, normalized (by subtraction) in terms of average preexposure accuracy, on the first 10 perexposure trials, the mean of the following 10 perexposure trials $(A)$, the mean of the last 10 perexposure trials $(B)$, and the 10 postexposure trials. Responses are in degrees as measured from the subject's nasum. Error bars represent \pm 1 standard error of the mean. 
ized with respect to preexposure performance. The elevator illusion is revealed by the markedly lower placement of the spot at the onset of hypergravity (Perexposure Test 1). Thus, subjects in both hypergravity conditions set the visual stimulus lower at the beginning of the hypergravity phase than during the 1-G preexposure phase because it appeared to be higher than it actually was. The apparent equality of the illusions for the two conditions on Perexposure Test 1 . which, it will be recalled, occurred prior to the initiation of the visual feedback trials, was to be expected because the two conditions had been treated in exactly the same manner to that point.

To examine the initial elevator illusion, the horizonsetting data were subjected to an order $(1 \mathrm{st} / 2 \mathrm{nd}) \times$ feedback (present/absent) $\times$ pre-per 1 ANOVA, in which preper 1 refers to the Preexposure Test versus Perexposure Test 1. Pre-per $1[F(1,9)=37.49, p<.01]$ and the order $\times$ feedback $[F(1,9)=5.14, p<.05]$ and the order $\times$ feedback $\times$ pre-per $1[F(1,9)=8.17, p<.05]$ interactions were all statistically significant. The very large $F$ for pre-per 1 (which accounted for $45.6 \%$ of the variance) confirms the existence of the elevator illusion for both conditions, whereas the absence of a main effect for feedback $[F(1,9)<1.0]$ supports the apparent equality in this regard between the two hypergravity conditions. Although the statistically significant three-way interaction was responsible for only $1 \%$ of the variance, it has potentially important theoretical implications, which will be discussed in a later section.
An order (Ist/2nd) $\times$ feedback (present/absent) ANOVA $\times$ test $(2-7)$ was computed on the data from Perexposure Tests $2-7$, the period during which the feedback variable was manipulated. According to this analysis, test $[F(5,45)=$ $9.01, p<.01]$ and feedback $[F(1,9)=7.08, p<.05]$ were statistically significant. Thus, both continued exposure to hypergravity and visual error-corrective feedback caused a decline in the elevator illusion, the latter effect having been predicted by the interaction hypothesis. The absence of a statistically significant perexposure test $\times$ feedback interaction indicates that the two effects were additive. Finally, an examination of Figure 2 reveals that the entire effect of visual feedback was present on the first test, a result to be discussed subsequently.

Adaptation to sensory rearrangement typically results in a negative aftereffect, usually measured by the difference between pre- and postexposure measures (see, e.g., Welch, 1978). As can be seen in Figure 3, only the visualfeedback condition produced a statistically significant aftereffect $[t(9)=1.97, p<.05$, one-tailed].

\section{Visual-Motor Coordination (Target Pointing)}

The results for the target-pointing measure can be seen in Figure 4. To reduce the complexity of this figure, the error bars for the 1-G data points (all of which overlapped the preexposure baseline) have been omitted. As expected, there was a tendency to reach too high in the no-visualfeedback condition and a suppression (albeit partial) of this tendency in the visual-feedback condition. Although

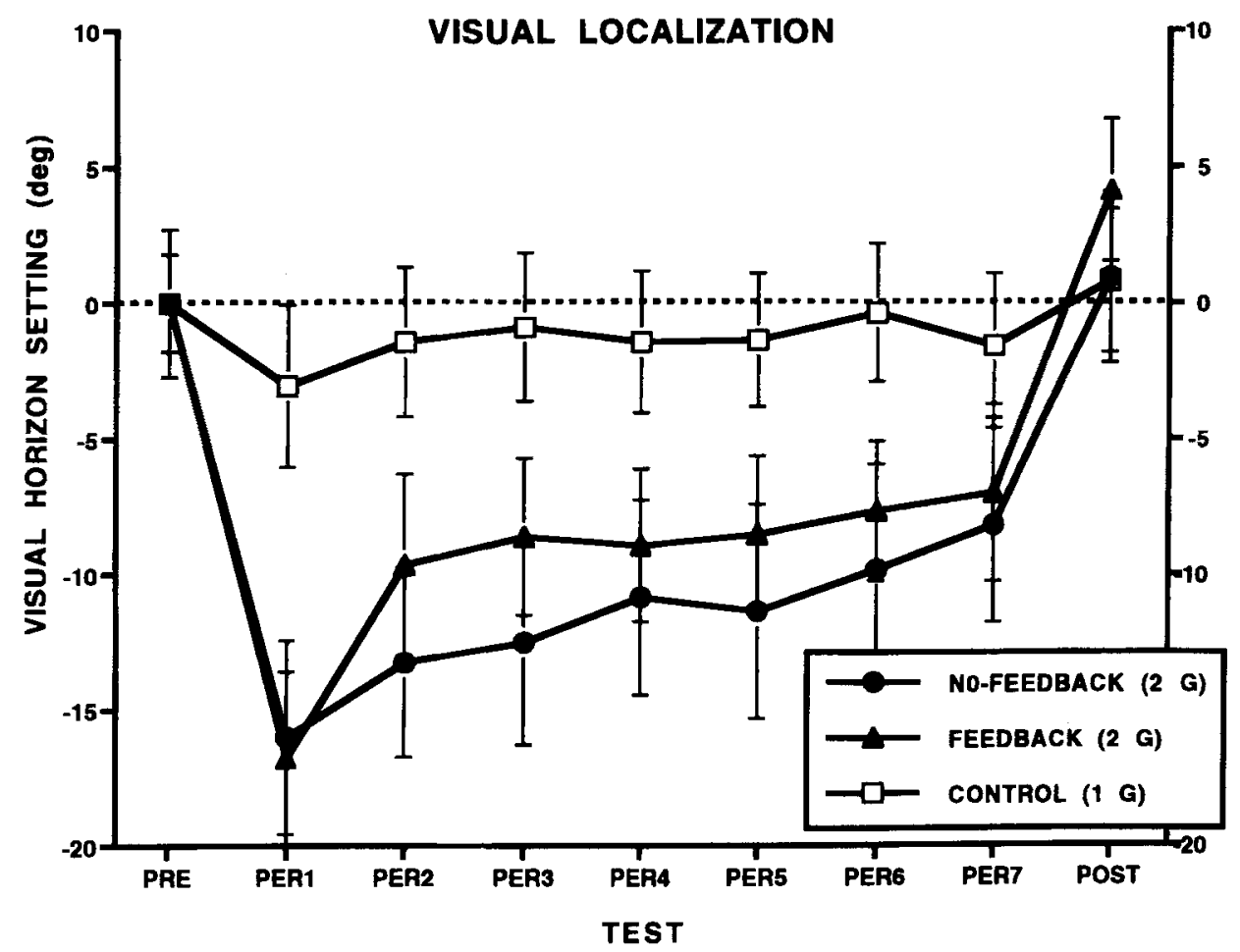

Figure 2. Visual horizon settings for visual-feedback, no-visual-feedback, and 1-G control conditions on the per- and postexposure measures, normalized (by subtraction) in terms of preexposure accuracy. Error bars represent \pm 1 standard error of the mean. 


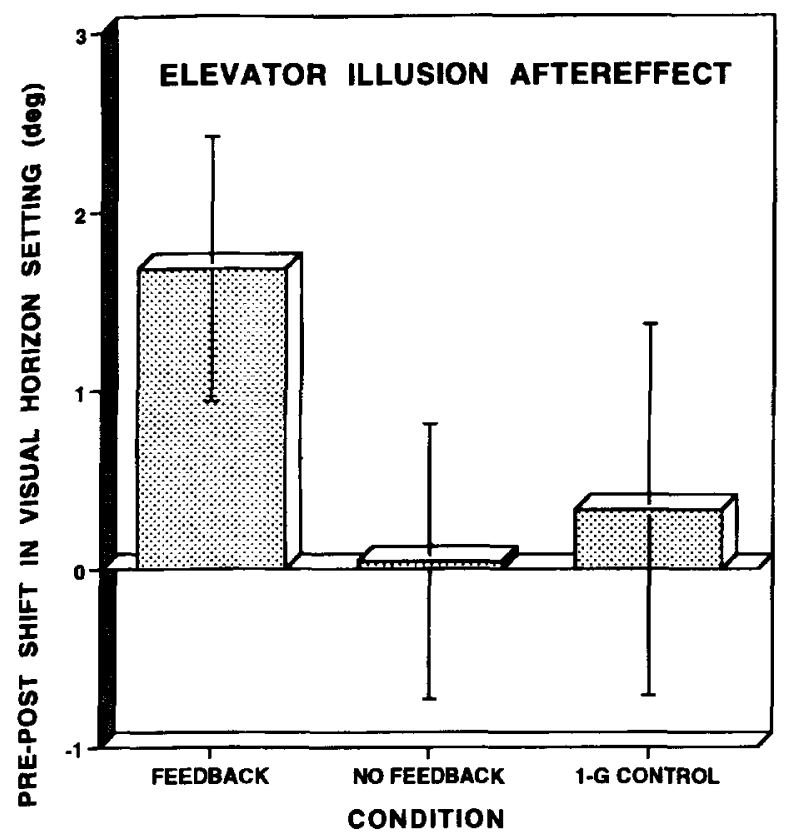

Figure 3. Pre-post shift in visual horizon settings for visualfeedback, no-visual-feedback, and 1-G control conditions. Error bars represent \pm 1 standard error of the mean.

an order $(1 \mathrm{st} / 2$ nd $) \times$ tests $(2-13) \times$ feedback (present $/$ absent) ANOVA failed to produce statistical significance for the factor of feedback $[F(1,9)=4.76, p>.05]$ (or any other factor), a correlated-groups $t$ test revealed a statistically significant difference between the two hypergravity conditions $[t(9)=4.48, p<.01]$.

It is important to observe that, contrary to our expectations, the magnitude of the target-pointing errors for the no-visual-feedback condition (or for both conditions on Perexposure Tests $I$ and 14 , on which visual feedback was denied) was much smaller than the elevator illusion measured for the same subjects. For example, whereas the initial elevator illusion averaged about $17^{\circ}$ (Figure 2), initial target-pointing error was only slightly over $2^{\circ}$ (Figure 4 ). The implications of this anomalous result are discussed in the next section.

\section{DISCUSSION AND CONCLUSIONS}

To our knowledge, this is the first report of a decline in the elevator illusion as a result of sustained hypergravity exposure. This finding probably stems from our use of a hypergravity exposure period that greatly exceeded that of any previously published studies (e.g., Cohen, 1973). An examination of Figure 2 suggests that if hypergravity had been further extended, the illusion would have continued to diminish. The fact that this decline occurred whether or not visual error-corrective feedback was provided suggests that it represents sensory habituation due to the mere presence of sustained hypergravity. Thus, it may be proposed that the continuous and vigorous stimulation of the otolith organs that occurred in both hypergravity conditions reduced their responsivity, which, in turn, led to a decline in neural outflow to the eye muscles and a concomitant attenuation of the elevator illusion. Whatever the underlying mechanism, and contrary to the suggestion in the introduction, it appears that passive exposure to hypergravity can lead to a substantial reduction of this vestibular-visual illusion, at least while the condition of excessive gravity persists.

Alternatively, the decrement obtained for both conditions might have been a visual manifestation of a change in felt eye position caused by the excessive neural outflow to the eye muscles required to fixate the visual targets during hypergravity exposure. This suggestion is a variant of the "eye muscle potentiation" hypothesis proposed by Ebenholtz and his colleagues (see, e.g., Ebenholtz, 1974; Ebenholtz \& Wolfson, 1975) as an alternative to the visual recalibration explanation of prism-adaptive changes in vision. According to this notion, if the eyes are held to one side for a while (e.g., to fixate a prismatically displaced target), they will feel more and more as if they have returned to their normal, straight-ahead resting position and perceived egocentric visual localization will follow suit. Likewise, when the prism is removed and the task is to fixate an object that is objectively straight ahead, the eyes will feel as if they are now turned in the direction opposite the previous prismatic displacement, causing the visual target to appear to be there as well; this, of course, is the negative aftereffect. Applying this conceptualization to the present situation, it could be argued that, during hypergravity exposure, subjects' eyes came to feel progressively less upwardly turned, causing apparent visual horizon to drift back toward preexposure levels. One problem with this explanation is that it would predict a postexposure negative aftereffect for the no-visual-feedback (as well as for the visual-feedback) condition. However, as we have seen (Figure 4), such an aftereffect appears to have occurred only for the visual-feedback condition.

The prediction from the interaction hypothesis that visual error-corrective feedback would attenuate the elevator illusion was supported, although the effect of this variable was much weaker than that of mere exposure (see Figure 2). This difference between the two hypergravity conditions may indicate the presence of the type of adaptive recalibration commonly assumed to be the basis of adaptation to other sensory rearrangements (see, e.g., Welch, 1978). The fact that only the visual-feedback condition produced a postexposure negative aftereffect, one of the hallmarks of prism adaptation, reinforces this interpretation.

It is important to note that the effect of visual feedback that was seen on the very first perexposure test failed to increase during the remainder of the exposure period (see Figure 2). Perhaps this outcome can be explained by the fact that subjects' initial target-pointing errors were much smaller than their elevator illusions and were therefore very quickly eliminated (see Figure 4). Thus, it is possible that if these errors had been commensurate with the large elevator illusion that these subjects experienced, the difference between the two curves (Figure 2) would have continued to grow during the exposure period. In any event, 


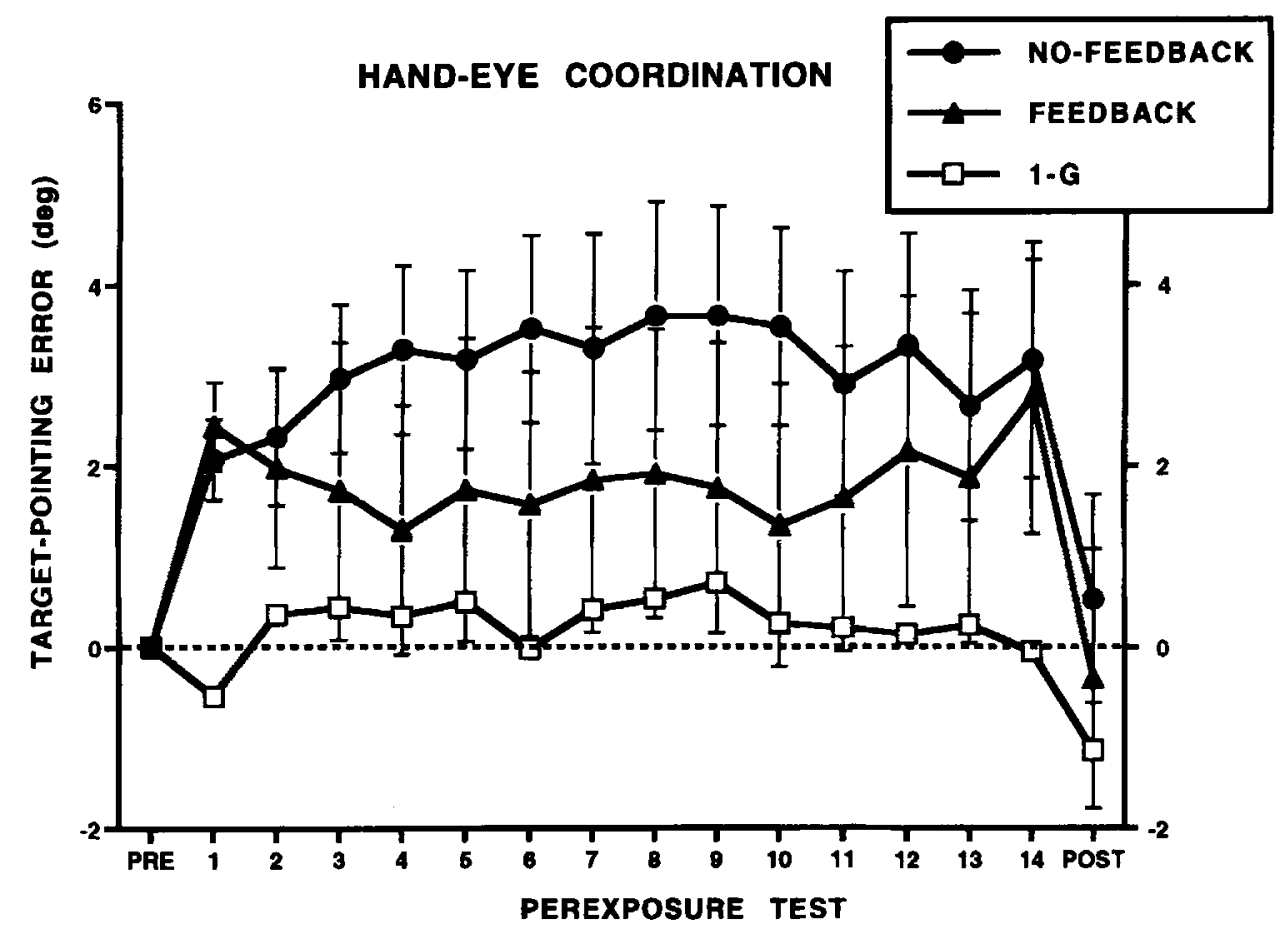

Figure 4. Target-pointing errors for visual-feedback, no-visual-feedback, and 1-G control conditions for per- and postexposure measures, normalized (by subtraction) in terms of preexposure accuracy. Error bars represent \pm 1 standard error of the mean.

the large discrepancy between visual localization and open-loop hand-eye coordination seen here casts serious doubt on our tacit assumption that the apparent upward displacement of visual targets in the elevator illusion represents a shift in egocentric direction tantamount to prismatic displacement (in the vertical dimension), where the two response measures usually produce equivalent errors (see, e.g., Welch, 1978).

One possible interpretation of our results is that the elevator illusion is composed of two separable components. The first of these is a change in the registered relationship of eye and head, which is the traditional way of conceptualizing the illusion. The second proposed component is a shift in the perceived relation of the body to gravity. The first of these should be accurately reflected in open-loop target pointing (assuming, as we have here, that the muscleloading effect has been eliminated). This would not be the case with the second component, however, because in this case there would probably be no change in the perceived location of the visual target relative to the body. Because visual target-pointing errors were so small relative to the elevator illusion, this second proposed component would seem to have played a much larger role in the illusion obtained in this experiment than the change in oculomotor control.

Such a two-component model might be applicable to the results of other studies of altered spatial orientation (e.g., pitched visual environments), in which visual judgments have revealed much stronger effects than the accompanying visual-motor behavior (e.g., Ballinger, 1988;
Nemire \& Cohen, 1993; Welch \& Post, in press). It is likely that future research will reveal still other bases for the elevator illusion.

It is not obvious how the eye muscle potentiation interpretation, discussed above, could explain the effect of visual feedback on the elevator illusion. However, perhaps because subjects in the visual-feedback condition saw both the finger and the target for $2 \mathrm{sec}$ on each target-pointing trial, the total time during which the eyes were turned upward was greater for them than in the no-visual-feedback condition, leading to greater eye muscle potentiation. One control for this possibility would be a $2-\mathrm{G}$ condition in which subjects did not point at targets, but merely held their eyes above eye level for a period equal to that of the visual-feedback condition.

Still another potential explanation of the effect of errorcorrective visual feedback on the elevator illusion is that it does not represent a change in perception (visual or gravitational) at all. That is, it might be that the error-corrective feedback received by subjects in the visual-feedback condition simply caused them to change their criterion for visual horizon, rather than their perception of it. With this possibility in mind, it is recommended that future studies of the effects of error-corrective feedback on the elevator illusion, as well as on other sensory distortions, use measures that can discriminate between perception and response bias.

The significant feedback $\times$ order $\times$ pre-per interaction for the initial elevator illusion described in the Results section (Figure 5) warrants further discussion. In order to 


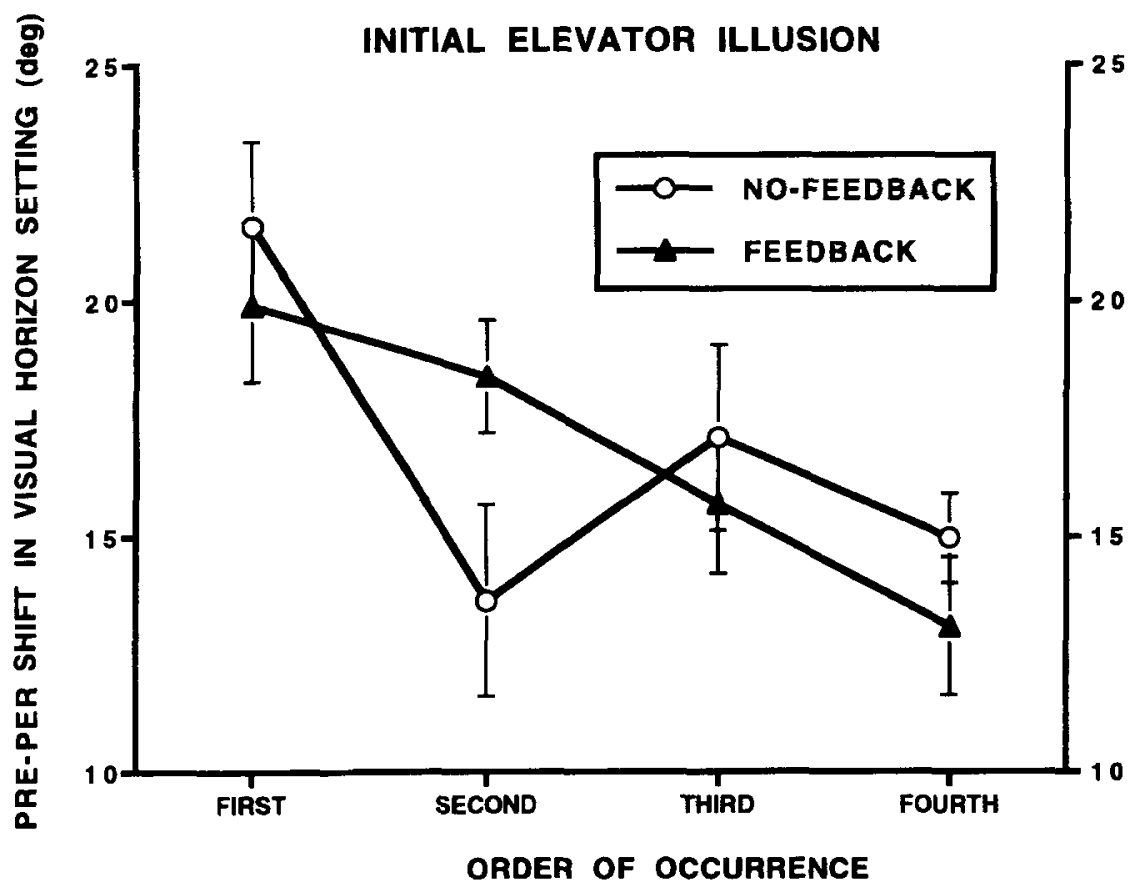

Figure 5. Pre-per shifts in visual horizon settings for visual-feedback and no-visualfeedback conditions presented according to order of testing session. Error bars represent \pm 1 standard error of the mean.

simplify Figure 5, the relationship was reduced to a twoway interaction by using the pre--per factor as the dependent variable (i.e., pre-per shift). This shift is, of course, the operational definition of the elevator illusion. It can be seen in the figure that the strength of the elevator illusion, as measured at the beginning of a given exposure phase (before the introduction of visual feedback), generally declined for both hypergravity conditions, from first to last session, with an interesting reversal between the second and third sessions for the no-visual-feedback condition. Visual examination of the data revealed no apparent change in preexposure horizon setting as a function of order, nor a difference in this regard between the two feedback conditions. Therefore, the decline in settings from first to fourth sessions represents a reduction in the initial effect of hypergravity on judgments of visual horizon (i.e., the elevator illusion as measured before sensory habituation and adaptation have had a chance to occur).

One interpretation of the pattern of results seen in Figure 5 is that the decrement in the elevator illusion in one session persists to the illusion measured at the outset of the next session (which, it will be recalled, usually occurred a week or more later). Furthermore, it would appear that the greater the reduction of the illusion in one session, the greater the later initial decrement. With respect to the visual-feedback condition, for example, the fact that the decline was modest when it occurred second (Figure 5) may be due to the fact that the session that preceded it entailed no visual feedback (i.e., the BAAB order) and thus presumably produced only sensory habituation. In contrast, when the no-visual-feedback condition occurred second, the very dramatic drop in the initial elevator illusion may have been because it was preceded by a visual-feedback condition (ABBA order), which we assume had produced both habituation and adaptation. Perhaps this double effect does not persist long enough to influence the illusion when the visual-feedback condition occurs third, although the illusion remains smaller than it was in the first session. There is thus suggestive evidence that subjects in this experiment acquired a "dual adaptation" (see, e.g., Cunningham \& Welch, 1994; Welch, Bridgeman, Anand, \& Browman, 1993) to the two conditions of excessive and normal gravity. This interpretation is, of course, quite speculative, and future studies specifically designed to examine it are in order.

To conclude, the facts that the elevator illusion can be substantially weakened by both sustained hypergravity exposure and error-corrective feedback, and that these effects persist for as long as several weeks, raise the possibility that other vestibularly induced illusions (oculogravic, posturogravic, oculogyral) can be similarly influenced.

\section{REFERENCES}

BALLINGER, C. J. (1988). The effects of a pitched field orientation on hand/eye coordination. Unpublished master's thesis, Naval Postgraduate School, Monterey, CA.

Bock, O., Howard, I. P., Money, K. E., \& Arnold, K. E. (1992). Accuracy of aimed arm movements in changed gravity. Aviation, Space, \& Environmental Medicine, 63, 994-998.

Clark, B., \& GRAYBIEL, A. (1949). Linear acceleration and deceleration as factors influencing nonvisual orientation during flight. Journal of Aviation Medicine, 20, 92-101. 
COHEN, M. M. (1970a). Hand-eye coordination in altered gravitational fields. Aerospace Medicine, 41, 647-649.

CoHEN, M. M. (1970b). Sensory-motor adaptation and aftereffects of exposure to increased gravitational forces. Aerospace Medicine, 41, 318-322.

CoHEN, M. M. (1973). Elevator illusion: Influences of otolith organ activity and neck proprioception. Perception \& Psychophysics, 14, 401-406.

CoHen, M. M. (1981). Visual-proprioceptive interactions. In R. D. Walk \& H. L. Pick (Eds.), Intersensory perception and sensory integration (pp. 175-215). New York: Plenum.

Cohen, M. M., Crosbie, R. J., \& Blackburn, L. H. (1973). Disorienting effects of aircraft catapult launchings. Aerospace Medicine, 44, 37-39.

COHEN, M. M., \& WELCH, R. B. (1988). Hand-eye coordination during and after parabolic flight. Aviation, Space, \& Environmental Medicine, 59, 68B.

COHEN, M. M., \& Welch, R. B. (1992). Visual-motor control in altered gravity. In L. Proteau \& D. Elliott (Eds.), Vision and motor control (pp. 153-175). New York: Elsevier.

Correia, M. J., Hixson, W. C., \& Niven, J. I. (1968). On predictive equations for subjective judgments of vertical and horizon in a force field. Acta Oto-Laryngologica, Monograph Supplement 230.

Cunningham, H. A., \& Welch, R. B. (1994). Multiple concurrent visual-motor mappings: Implications for models of adaptation. Journal of Experimental Psychology: Human Perception \& Performance, 20, 987-999.

DAY, R. H. (1962). The effects of repeated trials and prolonged fixation on error in the Mueller-Lyer figure. Psychological Monographs, 76, No. 14(Whole No. 533).

EBENHOLTZ, S. M. (1974). The possible role of eye muscle potentiation in several forms of prism adaptation. Perception, 3, 477-485.

Ebenholtz, S. M., \& Wolfson, D. M. (1975). Perceptual aftereffects of sustained convergence. Perception \& Psychophysics, 17, 485-491.

Festinger, L., White, C. W., \& Allyn, M. R. (1968). Eye movements and decrement in the Mueller-Lyer illusion. Perception \& Psychophysics, 3, 376-382.

GRAYBIEL, A. (1952). Oculogravic illusion. AMA Archives of Ophthalmology, 48, 605-615.

Graybiel, A., Clark, B., \& MacCorquodale, K. (1947). The illusory perception of movement caused by angular acceleration and by centrifugal force during flight. I. Methodology and preliminary results. Journal of Experimental Psychology, 7, 170-177.

Graybiel, A., \& Hupp, D. I. (1946). The oculo-gyral illusion, a form of apparent motion which may be observed following stimulation of the semicircular canals. Journal of Aviation Medicine, 17, 3-27.

HOENIG, P. (1972). The effects of eye movements, fixation and figure size on decrement in the Mueller-Lyer illusion. Unpublished doctoral dissertation, The New School for Social Research, New York.
Kalil, R. E., \& Freedman, S. J. (1966). Persistence of ocular rotation following compensation for displaced vision. Perceptual \& Motor Skills, 22, 123-126.

KEOWN, T. (1994, October 7). Driver Breedlove accelerates toward the sound barrier: Living in a fast lane that's his alone. San Francisco Chronicle.

KILPATRICK, F. P. (1954). Two processes in perceptual learning. Journal of Experimental Psychology, 47, 362-370.

Marcus, J. T., \& Van Holten, C. R. (1990). Vestibulo-ocular responses in man to $\mathrm{Gz}$ hypergravity. Aviation, Space, \& Environmental Medicine, 61, 631-635.

NemiRE, K., \& COHEN, M. M. (1993). Visual and somesthetic influences on postural orientation in the median plane. Perception \& Psychophysics, 53, 106-116.

SCHÖNE, H. (1964). On the role of gravity in human spatial orientation. Aerospace Medicine, 35, 764-772.

UhLARIK, J. J., \& CANON, L. K. (1971). Influence of concurrent and terminal exposure conditions on the nature of perceptual adaptation. Journal of Experimental Psychology, 91, 233-239.

VoN BECKH, H. J. A. (1959). Human reactions during flight to acceleration preceded by or followed by weightlessness. Aerospace Medicine, 30, 391-409.

WeLCH, R. B. (1978). Perceptual modification: Adapting to altered sensory environments. New York: Academic Press.

Welch, R. B., Bridgeman, B., Anand, S., \& Browman, K. E. (1993). Alternating prism exposure causes dual adaptation and generalization to a novel displacement. Perception \& Psychophysics, 54, 195-204.

WELCH, R. B., \& POST, R. B. (in press). Accuracy and adaptation of reaching in pitched visual environments. Perception \& Psychophysics.

Whiteside, T. C. D. (1961). Hand-eye coordination in weightlessness. Aerospace Medicine, 32, 719-725.

Whiteside, T. C. D., Graybiel, A. A., \& Niven, J. I. (1965). Visual illusions of movement. Brain, 88, 193-210.

\section{NOTES}

1. Negative $\mathrm{G}$ in the $x$-axis causes an even more dramatic drop in the visual field, as recently reported by $\mathrm{C}$. Breedlove when his droge chute brought his jet-powered car to an abrupt halt from a 600 -mph dash on the Utah salt flats.

2. For reasons that remain unclear, a recent study by Bock, Howard, Money, and Arnold (1992) failed to obtain the latter effect.

(Manuscript received October 10, 1994; revision accepted for publication June 14, 1995.) 